\title{
ANÁLISIS SOBRE EL CRIMEN DE AGRESIÓN EN LA CORTE PENAL INTERNACIONAL A PARTIR DE LA CONFERENCIA DE REVISIÓN (KAMPALA). RETOS Y PERSPECTIVAS
}

\author{
ANALYSIS OF THE CRIME OF AGGRESSION \\ IN THE INTERNATIONAL CRIMINAL \\ COURT FROM THE REVIEW CONFERENCE \\ (KAMPALA). CHALLENGES AND PROSPECTS
}

Gerardo A. Durango Álvarez*

PARA CITAR ESTE ARTículo / TO CITE THIS ARTIClE Durango Álvarez, G., Análisis sobre el crimen de agresión en la Corte Penal Internacional a partir de la Conferencia de Revisión (Kampala). Retos y perspectivas, 24 International Law, Revista Colombiana de Derecho Internacional, 193-218 (2014).

doi:10.11144/Javeriana.IL14-24.acac

* Doctor en Derecho: derechos fundamentales, Magíster en Filosofía del Derecho (Universidad Carlos III, Madrid). Profesor Titular de la Universidad Nacional de Colombia, sede Medellín, Departamento de Ciencia Política. Director del Grupo de Investigación Derechos Fundamentales y Teoría Política. Autor de los libros: La participación politica como derecho fundamental. Un análisis desde la democracia deliberativa (Universidad Nacional, Medellín, 2013); El principio discursivo y los derechos fundamentales en la teoría habermasiana (Editorial Española, Madrid, 2012). Inclusión y desarrollo de las acciones positivas en la jurisprudencia de la Corte Constitucional colombiana (Diké-Universidad Nacional, Medellín, 2011); Democracia deliberativa y derechos fundamentales (Temis, Bogotá D.C., 2006); Correo electrónico: gadurangoa@unal.edu.co 


\section{RESUMEN}

El crimen de agresión constituye uno de los actos más graves cometidos por un Estado contra otro, en tanto afecta, en primer lugar el derecho a la autodeterminación y soberanía del respectivo Estado; en segundo término, al hacer uso de la fuerza se comete una de las mayores infracciones contra el derecho internacional y la paz mundial. El crimen de agresión ha tenido un lento desarrollo por parte de la comunidad internacional, debido a las presiones políticas y económicas que han ejercido países con capacidad de influencia mundial como Estados Unidos, Rusia, China, Inglaterra y Francia, que han establecido definiciones y resoluciones a su amaño, tal como la consagrada en la Resolución 3314 de la onu de 1974. La comunidad internacional ha buscado, de forma reiterada, establecer una definición vinculante para los Estados e individuos que cometen este tipo de crimen, además de su sanción por la comisión del ilícito internacional, lo que ha sido imposible. Sin embargo, solo hasta la Primera Conferencia de Revisión de los Estados Partes del Estatuto de Roma (en adelante, ER) de la Corte Penal Internacional, celebrada en Kampala, Uganda, en el año 2010, se logró una histórica definición-consensuada por los Estados partes- sobre el crimen de agresión y la competencia en cabeza de la Corte Penal Internacional, que se espera entre en vigor después de que treinta Estados partes ratifiquen las enmiendas del Artículo 8(1)bis y del Artículo 15(3)bis del ER. El recorrido para llegar a esta definición no ha sido fácil, ante todo por el poder de veto de los miembros permanentes del Consejo de Seguridad de ver limitado el tan cuestionado derecho al uso de la fuerza tal y como está consagrado en el capítulo VII de la Carta de la ONU. Por tanto, este artículo pretende abordar las siguientes preguntas: ¿La nueva definición del crimen de agresión logrará un cambio en las decisiones unilaterales tomadas por los miembros permanentes del Consejo de Seguridad, de acuerdo con el capítulo VII de la onU, en tanto a las sanciones impuestas a algunos Estados?

Palabras clave autor: Crimen de agresión, acto de agresión, Corte Penal Internacional, Consejo de Seguridad, competencia.

Palabras clave descriptores: Agresión (Derecho internacional), Corte Penal Internacional, Naciones Unidas. Consejo de Seguridad, competencia económica. 


\section{ABSTRACT}

The crime of aggression is one of the most serious acts committed by one State against another because it affects the right to self-determination and sovereignty of the State concerned, and by means of force, is it making major infractions against the international law and world peace. Now, the crime of aggression has had slow development by the international community due to political and economic pressure exerted by influential countries as USA, Russia, China, England and France. These countries have cunningly established definitions and resolutions as in the 3314 un Resolution of 1974. The international community has repeatedly sought to provide a definition binding on states and individuals who commit this type of crime, besides the sanctions - impossible yet-for the commission of this international offense. However, only until the First Review Conference of States Parties to the Rome Statute of the International Criminal Court, held in Kampala, Uganda in 2010, achieved a historic-agreed-upon definition of the crime of aggression and the competence of his prosecution by the International Criminal Court, which is expected to enter into force after thirty States Parties ratify the amendment to Art. 8(1) bis of this Statute. The journey to get to this definition has not been easy or simple, first of all by the veto of the permanent members of the Security Council to see limited the still-questioned right to use force as is established in Chap. VII of the UN Charter. Therefore, this work aims to develop the following question: Will the new definition of the crime of aggression affect the powers of the Security Council under Chapter VII of the UN regarding the sanctions imposed to some States?

Keywords author: Crime of aggression, International Criminal Court, Security Council, competition, act of aggression.

Keywords plus: Aggression (International law), International Criminal Court, United Nations. Security Council, competition.

\section{SUMARIO}

I. LA CORTE PENAL Y EL CRIMEN DE AGRESIÓN.- II. EL CRIMEN DE AGRESIÓN, SU DEFINICIÓN Y COMPETENCIA DESPUÉS DE KAMPALA.- III. EL CRIMEN DE AGRESIÓN DESDE LA PERSPECTIVA DE LAS RELACIONES INTERNACIONALES. LA OPOSICIÓN DE LOS ESTADOS PERMANENTES DEL CONSEJO DE SEGURIDAD.- IV. CONCLUSIÓN.BiBLIOGRAFÍA. 


\section{La CORTE PENAL y El CRIMEN DE AgResión}

La Corte Penal Internacional es el resultado del desarrollo y combinación del derecho internacional, de los principios de derecho penal internacional -responsabilidad individual por conductas punibles-, de los tribunales ad hoc y del derecho internacional humanitario -Convenios de Ginebra de 1949 y Protocolos I y II-. En la práctica, lo que hace la Corte Penal Internacional (CPI) es retomar y reestructurar los principales fundamentos de los diferentes instrumentos internacionales, y adecuarlos según la evolución del derecho penal internacional.

Como resultado de esta larga tradición, en 1998 los Estados parte reunidos en Roma atribuyeron competencia material a la Corte Penal Internacional ${ }^{1}$, en cuanto a los crímenes de genocidio $^{2}$, lesa humanidad ${ }^{3}$, de guerra ${ }^{4}$ y agresión. Respecto del crimen de guerra, la Corte Penal enjuicia actualmente a líderes de Uganda, República Democrática del Congo ${ }^{5}$ y República Centroafricana (estos primeros casos fueron remitidos de forma

1 A enero de 2014, 121 Estados han ratificado el Estatuto de Roma. En: http://www.iccnow. org/documents/CICCFS-RatificationsbyRegion_121_sp.pdf

2 A los efectos del presente Estatuto se entenderá por genocidio, cualquiera de los actos perpetrados con la intención de destruir total o parcialmente a un grupo nacional, étnico, racial o religioso, entre estos: a) Matanza de miembros del grupo; b) Lesión grave a la integridad física o mental de los miembros del grupo; c) Sometimiento intencional del grupo a condiciones de existencia que hayan de acarrear su destrucción física, total o parcial; d) Medidas destinadas a impedir nacimientos en el seno del grupo; e) Traslado por la fuerza de niños del grupo a otro grupo.

3 Se entiende por lesa humanidad, cualquiera de los actos que se cometan como parte de un ataque generalizado o sistemático contra una población civil y con conocimiento de este ataque. Entre estos: asesinato, exterminio, violación, prostitución forzada, embarazo forzado, esclavitud sexual, esterilización forzada, deportación o traslado forzoso de población, encarcelación u otra privación grave de la libertad física, tortura y desaparición forzada de personas

4 A los efectos del presente Estatuto, se entiende por crímenes de guerra: a) Infracciones graves de los convenios de Ginebra de 12 de agosto de 1949, a saber: actos cometidos en contexto de conflictos armados internacionales; actos cometidos en contexto de conflictos armados que no sean de índole internacional; homicidio intencional; tortura, tratos inhumanos, causar sufrimientos intencionados, ayudar a servir en fuerzas enemigas; deportación o traslado ilegal, detención ilegal; toma de rehenes, ataques a la población civil, causar muerte a un no combatiente, perfidia, atacar bienes prohibidos, saqueo, emplear armas prohibidas, mutilaciones; esclavitud sexual, prostitución, forzada, embarazo forzado, esterilización forzada y cualquier otra forma de violencia sexual.

5 En un fallo histórico, junio de 2012, la Corte Penal Internacional dictó sentencia condenatoria de 14 años de prisión en contra de Thomas Lubanga. 
directa por los mismos Estados a la CPI); ha iniciado investigaciones en Kenia y abierto una investigación preliminar en Colombia -en estos dos países el fiscal ha actuado motu proprio-, Afganistán, Honduras, Costa de Marfil e Israel.

En relación con los crímenes remitidos por el Consejo de Seguridad a la CPI, existen dos situaciones que cursan hoy ante la Corte: el caso de Darfur (Resolución 1593 de 2005) y Libia (Resolución 1970 de 2011). El Estatuto de Roma de 1998 de la Corte Penal Internacional estipuló el crimen de agresión como un delito de trascendencia internacional, y en principio le atribuyó una enunciación formal en el Artículo 5(d):

"Crímenes de la competencia de la Corte

1. La competencia de la Corte se limitará a los crímenes más graves de trascendencia para la comunidad internacional en su conjunto. La Corte tendrá competencia, de conformidad con el presente Estatuto, respecto de los siguientes crimenes:

a) El crimen de genocidio;

b) Los crimenes de lesa humanidad;

c) Los crímenes de guerra;

d) El crimen de agresión".

Respecto al crimen de agresión, el ER pospuso lo relativo a la definición y competencia "ratione materiae" -aunque sí lo formuló para los otros crímenes, lo que dejó una especie de laguna jurídica-, hasta que la Asamblea de los Estados partes aprueben las disposiciones normativas que lo desarrollen, según el Artículo 5(2) que dice: "La Corte ejercerá competencia respecto del crimen de agresión una vez que se apruebe una disposición de conformidad con los artículos 121 y 123 en que se defina el crimen y se enuncien las condiciones en las cuales lo hará. Esa disposición será compatible con las disposiciones pertinentes de la Carta de las Naciones Unidas" (este apartado fue derogado por el Artículo 8 bis del ER).

En relación con este crimen, es de precisar que la comunidad internacional no había logrado hasta la fecha -hasta el consenso logrado por de los Estados partes en Kampala, en 2010- una 
definición ${ }^{6}$ jurídica inclusiva para todos los Estados sobre el crimen de agresión, la cual estableciera responsabilidad penal internacional por parte de quien comete la conducta punible, esto es, un individuo o una pluralidad de personas que actuaran con apoyo directo o indirecto del respectivo Estado y sus líderes. En tal sentido, en este ensayo interesa analizar el crimen de agresión ${ }^{7}$ y su definición a partir de la Conferencia de Revisión de Kampala y sus implicaciones para la comunidad internacional.

Por consiguiente, puede decirse que el crimen de agresión presenta una evolución de varias décadas en la comunidad internacional, pero sin una definición, competencia y jurisdicción clara y precisa. Cabe destacar que en los juicios de Nüremberg ${ }^{8}$ y Tokio ${ }^{9}$, este tipo de delito -además del de guerra y de lesa humanidad-fue atribuido de forma unilateral por parte de las potencias triunfadoras a los militares de los países vencidos en la Segunda Guerra Mundial. Se acusa que fueron juicios penales con mínimas garantías procesales, en que se desconocieron principios elementales como el de nullum crimen sine lege, situación que desencadenó la condena a muerte y cadena perpetua para muchos de los militares enjuiciados. Este tipo de procesos impuestos por las potencias vencedoras son, según Kai Ambos,

6 La definición fue preparada por el grupo especial de trabajo sobre el crimen de agresión. Ver al respecto el documento: Report of the Special Working Group on the Crime of Aggression. Doc. ICC-ASP/7/SWGCA/2. En www.icc-cpi.int/NR/rdonlyres/23C70E38-C4134CBE-8F53-1BD15E142CCE/140910/ICCASP4SWGCA1_final_spanish.pdf (2009).

7 Los fundamentos normativos sobre el crimen de agresión se encuentran consagrados en el Artículo 1, párrafo 1 de la Carta de la onU, donde se establece de manera general y formal que uno de los propósitos fundamentales de la onv es: "Mantener la paz y la seguridad internacionales, y con tal fin: tomar medidas colectivas eficaces para prevenir y eliminar amenazas a la paz, y para suprimir actos de agresión u otros quebrantamientos de la paz". Además se encuentra en las siguientes resoluciones de la onU: Resolución 95 (I) de 11 de diciembre de 1946; Resolución 377 (V) de 3 de noviembre de 1950; Resolución 2131 (XX) de 21 de diciembre de 1965; Resolución 2625 (XXV) de 24 de octubre de 1970; Resolución 3314 (XXIX) de 14 de diciembre de 1974; Sentencias de los tribunales de Nüremberg y Tokio (artículos 5 y 6); Artículo 8bis de la Primera Conferencia de Revisión -enmiendadel Estatuto de Roma en Kampala, Uganda.

8 Este tipo de tribunales impuso a los vencidos un derecho punitivo inquisitivo, esto es, el Tribunal de Nüremberg tipificó cuatro cargos en contra de los alemanes: crímenes contra la paz (el cual surge por primera vez en el derecho internacional penal, como crimen de agresión), crímenes contra la humanidad (genocidio), crímenes de guerra y conspiración.

9 A los japoneses se les juzgó por crímenes de guerra. 
una "imposición (vertical) del sistema ad hoc de la justicia penal internacional"10.

Así, dentro del establecimiento de los tribunales más importantes, conforme al Consejo de Seguridad de la onU -capítulo VII-, se creó en 1993 el Tribunal Internacional ad hoc para la ex Yugoslavia -Resolución 827 de 25 de mayo de 1993-, con el fin de juzgar los crímenes de guerra y genocidio cometido en los Balcanes. Por medio de este tribunal, se juzgó y condenó a los perpetradores de crímenes de guerra, genocidio y crímenes contra la humanidad; todos ellos tipificados como infracciones graves a la Convención de Ginebra de 1949.

Por su parte, la Resolución 995 de 1994 del Consejo de Seguridad de las Naciones Unidas, creó el Tribunal Internacional para Ruanda destinado a procesar por vía legal a los responsables del genocidio y otras violaciones en contra del DIH, cometidos durante el conflicto racial interno entre Hutus y Tutsis.

\section{EL CRIMEN DE AGRESIÓN, SU DEFINICIÓN Y COMPETENCIA DESPUÉS DE KAMPALA}

La Primera Conferencia de Revisión de los Estados tenía como propósito revisar y adoptar una propuesta conjunta sobre la definición del crimen de agresión, según lo dispuesto en el Artículo 123 del ER. Lo importante de esta reunión entre los Estados partes de la Corte Penal Internacional fue que, por consenso, se logró una definición sobre el crimen de agresión; sobre el crimen y sobre el acto de agresión, en el Artículo 8 (1) y (2) bis de la siguiente manera: "A los efectos del presente Estatuto, una persona comete un 'crimen de agresión' cuando, estando en condiciones de controlar o dirigir efectivamente la acción política o militar de un Estado, dicha persona planifica, prepara, inicia o realiza un acto de agresión que por sus características, gravedad

$10 \mathrm{~K}$. Ambos, El derecho penal internacional en la encrucijada: de la imposición ad hoc a un sistema universal basado en un tratado internacional, en Future Perspectives on International Criminal Justice, 161 (C. Stahn \& Van Den Herlk, Eds., T.M.C. Asser Press, The Hague, 2010). 
y escala constituya una violación manifiesta de la Carta de las Naciones Unidas".

Así, con respecto al crimen de agresión, la definición conjuga los verbos rectores "planifica", "prepara", "inicia", "realiza"; con este último se establece que el crimen de agresión ha iniciado o se ha llevado cabo, esto es, se ha ejecutado la conducta punible de agresión conforme artículo mencionado, lo que suprime la posibilidad de interpretación. Sostiene Kai Ambos: "La tentativa, como forma autónoma de responsabilidad, nunca ha desempeñado ningún rol en los procedimientos penales internacionales"11.

Se colige de la afirmación anterior, que si la Corte se dedicara a juzgar tentativas respecto del crimen de agresión, a quien planifica o prepara un acto de agresión, pero sin consumarlo, llevaría a la Corte a un callejón sin salida y le restaría credibilidad en la comunidad internacional. Por otro lado, vale la pena señalar que este tipo de crimen vincula como agente activo a los líderes políticos, religiosos o militares de un Estado, sea en su carácter individual o plural ${ }^{12}$.

En este contexto, el crimen de agresión remite de modo directo al Artículo 25, párrafo 3 bis del ER que señala claramente las características de la(s) persona(s) responsables de cometer el crimen de agresión -en tal situación se está ante un tipo especial como lo son los militares o dirigentes políticos de un Estado-, en tal sentido es claro el Artículo 25-3 bis al indicar que: "Por lo que respecta al crimen de agresión, las disposiciones del presente artículo solo se aplicarán a las personas en condiciones de controlar o dirigir efectivamente la acción política o militar de un Estado". A reglón seguido, define el acto de agresión en términos retomados de la Resolución de la Asamblea General de Naciones Unidas No. 3314 (XXIX), de 14 de diciembre de 1974:

11 K. Ambos, El crimen de agresión después de Kampala, 157 (Universidad Externado de Colombia, Bogotá D. C., 2012).

12 M. Zapico, El crimen de agresión y la Corte Penal Internacional, en Derecho penal entre la guerra y la paz, 722-723 (A. Nieto Martín \& S. Manacorda, Eds., Universidad Castilla de la Mancha, Madrid, 2009). 
"A los efectos del párrafo 1, por "acto de agresión” se entenderá el uso de la fuerza armada por un Estado contra la soberanía, la integridad territorial o la independencia política de otro Estado, o en cualquier otra forma incompatible con la Carta de las Naciones Unidas. De conformidad con la Resolución 3314 (XXIX) de la Asamblea General de las Naciones Unidas, de 14 de diciembre de 1974, cualquiera de los actos siguientes, independientemente de que haya o no declaración de guerra, se caracterizará como acto de agresión:

a) La invasión o el ataque por las fuerzas armadas de un Estado del territorio de otro Estado, o toda ocupación militar, aún temporal, que resulte de dicha invasión o ataque, o toda anexión, mediante el uso de la fuerza, del territorio de otro Estado o de parte de él;

b) El bombardeo, por las fuerzas armadas de un Estado, del territorio de otro Estado, o el empleo de cualesquiera armas por un Estado contra el territorio de otro Estado;

c) El bloqueo de los puertos o de las costas de un Estado por las fuerzas armadas de otro Estado;

d) El ataque por las fuerzas armadas de un Estado contra las fuerzas armadas terrestres, navales o aéreas de otro Estado, o contra su flota mercante o aérea;

e) La utilización de fuerzas armadas de un Estado, que se encuentran en el territorio de otro Estado con el acuerdo del Estado receptor, en violación de las condiciones establecidas en el acuerdo o toda prolongación de su presencia en dicho territorio después de terminado el acuerdo;

f) La acción de un Estado que permite que su territorio, que ha puesto a disposición de otro Estado, sea utilizado por ese otro Estado para perpetrar un acto de agresión contra un tercer Estado;

g) El envío por un Estado, o en su nombre, de bandas armadas, grupos irregulares o mercenarios que lleven a cabo actos de fuerza armada contra otro Estado de tal gravedad que sean equiparables a los actos antes enumerados, o su sustancial participación en dichos actos".

Como se aprecia de lo anterior, la interpretación y análisis del Artículo 8 bis(1), no puede hacerse de forma aislada del Artículo 8 bis(2), el cual desarrolla los elementos del acto de agresión y, por ende, las situaciones por las cuales un Estado comete acto de agresión contra otro Estado. Ahora bien, el acto de agresión está relacionado directamente con el crimen de agresión establecido en el Artículo 8 bis(1). Una pregunta conexa que se puede hacer sobre los actores no estatales que participan en el 
acto de agresión es ¿esta definición del Artículo 8 bis(2) incluye la participación de aquellos actores no estatales -grupos terroristas- que cometen crímenes de agresión? La respuesta de Kai Ambos es que no es recomendable, pues la inclusión de actores no estatales, modificaría la esencia de la definición ${ }^{13}$.

El acto de agresión, en la definición de Kampala, se encuentra íntimamente relacionado con el control sobre el uso de la fuerza establecido por el Consejo de Seguridad de la ONU, capítulo VII. En este sentido, el acto de agresión está asociado a la acción que cometa un Estado contra otro, ligado al sujeto activo que comete la conducta punible en tanto líder con capacidad de realzar el acto de agresión, tipificado en los Artículo 8 bis(1) y 25 (3) bis del ER. Se añade a esta definición que el ataque, de acuerdo con su gravedad y escala, no puede ir contra los postulados del capítulo VII mencionado, por tanto, es claro que retomaron aquí los conceptos de la Resolución 3314 de 19744.

En esta Resolución 3314 se entiende por agresión: "El uso de la fuerza armada por un Estado contra la soberanía, la integridad territorial o la independencia política de otro Estado, o en cualquier otra forma incompatible con la Carta de las Naciones Unidas". En cuanto al acto de agresión, la norma señala lo siguiente: "El primer uso de la fuerza armada por un Estado en contravención de la Carta constituirá prueba prima facie un acto de agresión".

Esta enunciación clásica es cuestionada por Andreas Paulus: "La Resolución 3314 no fue creada para apoyar el derecho penal; su fin consistió en validar las actuaciones del Consejo de Seguridad de la oNU en cuanto al acto de agresión conforme al capítulo VII"15.

13 K. Ambos, Óp. cit., p. 147.

14 Como afirma Salmón: "De esta forma, las características esenciales del crimen de agresión se configuran en el primer párrafo del artículo 8 bis y, el segundo, apunta más bien a una adecuada coordinación entre la responsabilidad estatal e individual. Constituye una suerte de puente entre ambas consecuencias de un mismo hecho lo que garantiza consistencia a la propia configuración del crimen de agresión y, de manera general, al Derecho internacional. E. Salmón, El crimen de agresión después de Kampala: soberanía de los Estados y lucha contra la impunidad, 45 (Instituto de Democracia y Derechos Humanos de la Pontificia Universidad Católica del Perú, Lima, 2011).

15 P. Andreas, Second Thoughts on the Crime of Aggression, 20 The European Journal of International Law, 4, 1117- 1128 (2009). Respecto de la vinculación de grupos terroristas en la definición de agresion, dice el autor: "The definitions contained in the resolution 3314 of 1974 definition of aggression, the Working Group has failed to endorse an extension of 
Por consiguiente, la Carta de las Naciones Unidas incluye el uso de la fuerza como legítimo ${ }^{16}$ según sus propios intereses, como se desprende de la capacidad de veto de los miembros permanentes del Consejo de Seguridad. En sus resoluciones, este consejo no considera a los restantes países miembros de la Organización, de allí la importancia de la definición, el consenso obtenido por los Estados Parte y la competencia del crimen de agresión en cabeza de la Corte Penal Internacional.

Conforme a las nuevas enmiendas hechas al ER (ver Tabla 1), le corresponde a la Sala de Cuestiones Preliminares aprobar el inicio de la investigación por un crimen de agresión, mediante votación mayoritaria de los jueces que la integran. El Artículo 15bis (7-8) limita la competencia exclusiva que hasta la fecha ostentaba el Consejo de Seguridad de la onu respecto al acto de agresión. Para una mejor compresión y posibilidad de comparar lo introducido en la enmienda, se presenta a continuación un cuadro comparativo entre la Resolución 3314 y el Artículo 8 bis sobre el crimen de agresión, aprobado en la Revisión de Kampala.

En este contexto y según la individualización de la conducta punible, es de aclarar que la penalización por la realización del crimen de agresión, sigue lo consagrado en el Artículo 77 del ER de la Corte Penal, donde se establecen diferentes tipos de pena: La Corte podrá, con sujeción a lo dispuesto en el Artículo 110, imponer a la persona declarada culpable de uno de los crímenes a que se hace referencia en el Artículo 5 del presente Estatuto una de las penas siguientes: reclusión que no exceda los 30 años; reclusión a perpetuidad cuando lo justifiquen la extrema gravedad del crimen y las circunstancias personales del condenado. Además la Corte podrá imponer multas con arreglo a los cri-

the notion of aggression to the voluntary support for terrorist groups in launching attacks against other states (Article 3(g). Thus, Resolution 3314 appears singularly unhelpful in solving these difficult issues".

16 Esto conforme el Artículo 24 de la Carta de la onU, al establecer que a fin de asegurar acción rápida y eficaz por parte de las Naciones Unidas, sus miembros confieren al Consejo de Seguridad la responsabilidad primordial de mantener la paz y la seguridad internacionales, y reconocen que el Consejo de Seguridad actúa en nombre de ellos al desempeñar las funciones que le impone aquella responsabilidad. 


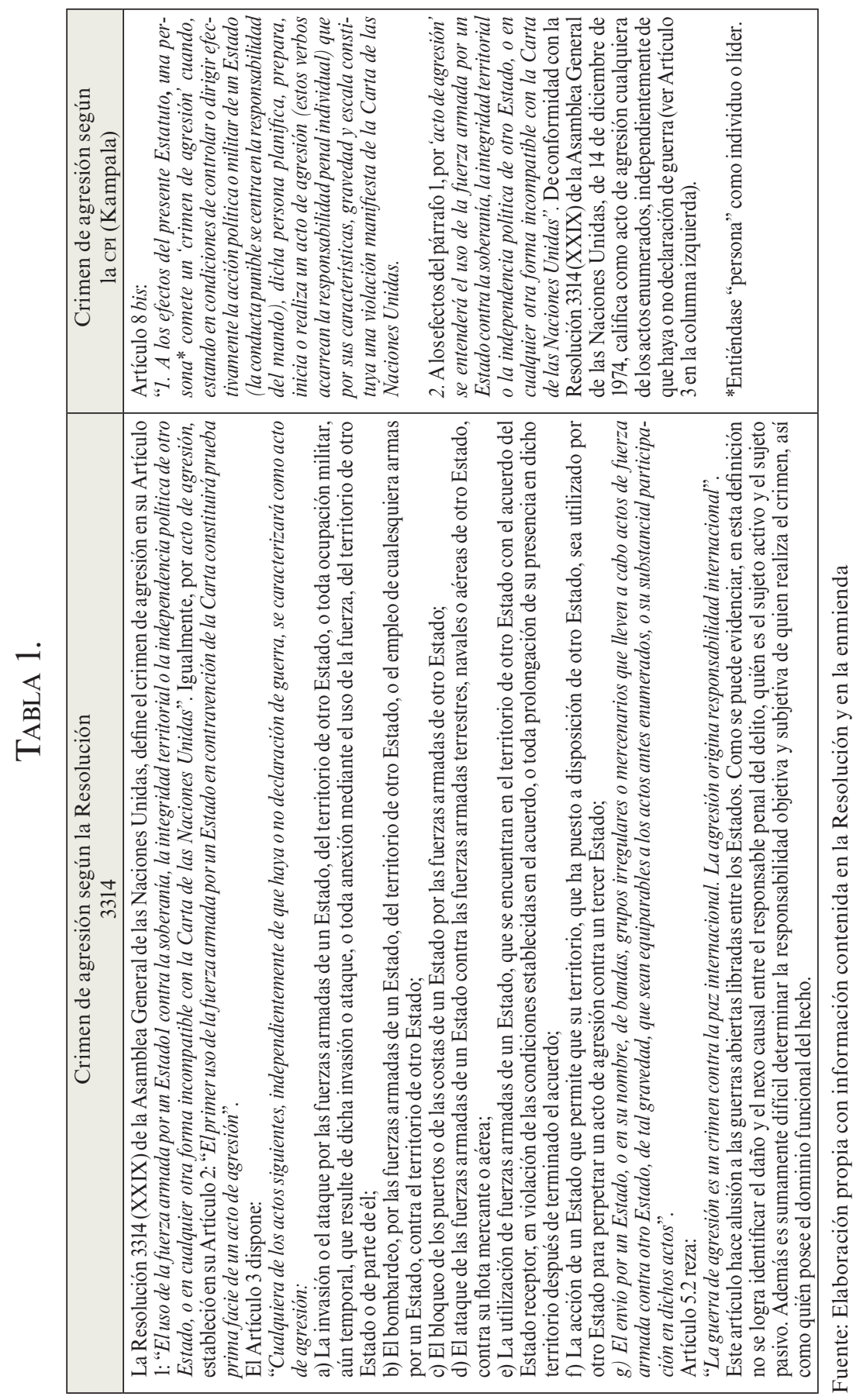


terios enunciados en las reglas de Procedimiento y Prueba; o el decomiso del producto, los bienes y los haberes procedentes, de forma directa o indirecta por la comisión del delito, sin perjuicio de los derechos de terceros de buena fe.

En cuanto a la competencia sobre los crímenes, estipulados en el Artículo 5 del ER, se debe hacer una lectura conjunta con el Artículo 12(1), condiciones previas para el ejercicio de la competencia, el cual menciona: "El Estado que pase a ser Parte en el presente Estatuto acepta por ello la competencia de la Corte respecto de los crímenes a que se refiere el artículo 5". En aras a la precisión, se debe decir que este apartado a su vez remite al Artículo 13 cuando indica que en el Ejercicio de la competencia:

"La Corte podrá ejercer su competencia respecto de cualquiera de los crímenes a que se refiere el artículo 5 de conformidad con las disposiciones del presente Estatuto si: a) Un Estado Parte remite al Fiscal, de conformidad con el artículo 14, una situación en que parezca haberse cometido uno o varios de esos crímenes;

b) El Consejo de Seguridad, actuando con arreglo a lo dispuesto en el Capítulo VII de la Carta de las Naciones Unidas, remite al Fiscal una situación en que parezca haberse cometido uno o varios de esos crímenes; o c) El Fiscal ha iniciado una investigación respecto de un crimen de ese tipo de conformidad con lo dispuesto en el artículo 15".

El Artículo 15bis del Estatuto de Roma, después de la enmienda, estableció la competencia de la Corte de la siguiente manera:

"Ejercicio de la competencia respecto del crimen de agresión

(Remisión por un Estado, motu proprio)

1. La Corte podrá ejercer su competencia respecto del crimen de agresión de conformidad con los apartados a) y c) del artículo 13, con sujeción a las disposiciones de este artículo.

2. La Corte únicamente podrá ejercer su competencia respecto de crímenes de agresión cometidos un año después de la ratificación o aceptación de las enmiendas por treinta Estados Partes.

3. La Corte ejercerá su competencia respecto del crimen de agresión de conformidad con el presente artículo, a condición de que se adopte una 
decisión después del $1^{\circ}$ de enero de 2017 por la misma mayoría de Estados Partes que se requiere para la aprobación de una enmienda al Estatuto ${ }^{17}$. 4. La Corte podrá, de conformidad con el artículo 12, ejercer su competencia sobre un crimen de agresión, resultante de un acto de agresión cometido por un Estado Parte, salvo que ese Estado Parte haya declarado previamente que no acepta esa competencia mediante el depósito de una declaración en poder del Secretario.

5. Respecto de un Estado no Parte en el presente Estatuto, la Corte no ejercerá su competencia respecto del crimen de agresión cuando éste sea cometido por los nacionales de ese Estado o en el territorio del mismo.

6. El Fiscal, si llegare a la conclusión de que existe fundamento razonable para iniciar una investigación sobre un crimen de agresión, verificará en primer lugar si el Consejo de Seguridad ha determinado la existencia de un acto de agresión cometido por el Estado de que se trate. El Fiscal notificará al Secretario General de las Naciones Unidas la situación ante la Corte, adjuntando la documentación y otros antecedentes que sean pertinentes.

7. Cuando el Consejo de Seguridad haya realizado dicha determinación, el Fiscal podrá iniciar la investigación acerca de un crimen de agresión.

8. Cuando no se realice dicha determinación en el plazo de seis meses desde la fecha de notificación, el Fiscal podrá iniciar los procedimientos de investigación respecto de un crimen de agresión, siempre y cuando la Sección de Cuestiones Preliminares, de conformidad con el procedimiento contenido en el artículo 15, haya autorizado el inicio de la investigación sobre un crimen de agresión, y el Consejo de Seguridad no haya decidido lo contrario de conformidad con el artículo 16.

9. La determinación de que hubo acto de agresión realizada por un órgano ajeno a la Corte no irá en perjuicio de las propias conclusiones de la Corte en virtud del presente Estatuto.

10. El presente artículo se entenderá sin perjuicio de las disposiciones correspondientes al ejercicio de la competencia respecto de otros crímenes a los que se hace referencia en el artículo 5.

Los numerales 4 y 5 del Artículo 15bis, permiten realizar el siguiente análisis:

a) En cuanto al ejercicio de la competencia respecto del crimen de agresión (remisión por un Estado, motu proprio, dice el numeral 4 del Artículo 15bis:

17 Este numeral fue aprobado por las presiones de EE UU como una forma de dilatar la entrada en vigencia del crimen de agresión. 
"La Corte podrá, de conformidad con el artículo 12, ejercer su competencia sobre un crimen de agresión, resultante de un acto de agresión cometido por un Estado Parte, salvo que ese Estado Parte haya declarado previamente que no acepta esa competencia mediante el depósito de una declaración en poder del Secretario. La retirada de esa declaración podrá efectuarse en cualquier momento y será considerada por el Estado Parte en un plazo de tres años".

Este numeral posibilita que los Estados realicen una declaración de no aceptación de la competencia de la Corte, esto es, una exención u opting out ${ }^{18}$ (optar por no). El peligro de esta excepción estribaría en la posibilidad de abrir una fisura que obstaculice la entrada en vigencia de la competencia de la Corte Penal, pues los Estados se pueden refugiar en este artículo para no ratificar la enmienda, como por ejemplo, Francia e Inglaterra. Ante esta situación, una de las salidas podría ser que el mismo Consejo de Seguridad remita el caso ante la CPI, lo que dejaría sin efecto la excepción de este numeral 4. Incluso, algunos países partes del ER.

b) En relación con el numeral 5 del Artículo 8bis, confirma que la Corte no puede ejercer la competencia sobre un crimen de agresión cuando sea cometido por nacionales del mismo Estado o de un Estado no parte, por tanto, quedaría como única opción la remisión del Consejo de Seguridad a la Corte. Con base en esto, se concedería un privilegio ${ }^{19}$ a aquellos Estados Permanentes del Consejo de Seguridad de la onu que no son parte de la Corte Penal Internacional (como Estados Unidos, Rusia y China), pues la enmienda no les afecta - esto iría en contra de los intereses de Francia e Inglaterra que sí son parte del Estatuto.

Lo más probable es que estos Estados veten alguna resolución que sea contraria a su interés, de manera que no operaría la remisión del Consejo a la Corte. Otros Estados que podrían salir beneficiados de esta situación son Israel, India e Irán que

18 Sobre este tema puede consultarse a C. Quesada Alcalá, El crimen de agresión como amenaza a la seguridad global, Cuadernos de estrategia, 160, 77-116 (2013).

19 K. Ambos, Óp. cit., p. 170. 
tampoco integran la CPI. En el caso de Francia e Inglaterra, por ser Estados partes, la Corte sí tiene competencia sobre la realización del crimen de agresión conforme a la territorialidad según el Artículo 12(2) del ER. Esto podría motivar a que estos países decidan no aceptar o ratificar la enmienda.

En lo atinente al conocimiento previo del crimen de agresión, por parte del Consejo de Seguridad, el ER y su modificación, se atribuye, conforme a la enmienda, una facultad previa a este organismo, para que determine previamente - por seis meses-la realización de un acto de agresión por parte de un Estado; esto conforme reza el Artículo 15bis, pár. 6 al decir:

"El Fiscal, si llegare a la conclusión de que existe fundamento razonable para iniciar una investigación sobre un crimen de agresión, verificará en primer lugar si el Consejo de Seguridad ha determinado la existencia de un acto de agresión cometido por el Estado de que se trate. El Fiscal notificará al Secretario General de las Naciones Unidas la situación ante la Corte, adjuntando la documentación y otros antecedentes que sean pertinentes".

Como puede colegirse, esta determinación previa de la existencia de un crimen de agresión es un primer filtro realizado por el Consejo de Seguridad conforme al capítulo VII de la Carta de onU. Este filtro jurisdiccional fue una incidencia ${ }^{20}$ y presión directa de Estados Unidos en la determinación previa del crimen de agresión por parte del Consejo de Seguridad, así como en la decisión que se adopte después del año 2017. Ahora bien, la determinación previa por parte del Consejo de Seguridad no es un impedimento para que la Corte llegue a sus propias conclusiones, en consonancia con el párrafo 9 del Artículo 8bis: "La determinación de que hubo acto de agresión realizada por un órgano ajeno a la Corte no irá en perjuicio de las propias conclusiones de la Corte en virtud del presente Estatuto".

20 H. Koh \& S. Rapp afirman: "U.S. national can be prosecuted for aggression so long as the $U . S$. remains a non-state party. And if we were to become a state party, we'd still have the option to opt out from having our nationals prosecuted for aggression. So we ensure total protection for our Armed Forces and other U.S. nationals going forward". Ver: Engagement in the International Criminal Court and the Outcome of the ICC Assembly of States Parties Conference. En www.state.gov/j/gcj/us_releases/remarks/2010/143178.htm (June 15, 2010). 
Este filtro inicial, según Ambos, reconcilia las posturas en conflicto respecto de la competencia del crimen de agresión: "Aquellas que no querían renunciar a la autoridad del Consejo de Seguridad (especialmente sus miembros permanentes), y aquellas que querían asegurar la integridad y autonomía de la Corte con base en las reglas ordinarias (especialmente las del art. 15). De hecho, el compromiso ciertamente alcanzó el máximo realista (y al mismo tiempo inesperado) en términos de asegurar la independencia de la Corte"21.

III. El CRIMEN DE AGRESIÓN DESDE LA PERSPECTIVA DE LAS RELACIONES INTERNACIONALES. LA OPOSICIÓN DE LOS Estados Permanentes del Consejo de Seguridad

Desde un punto de vista analítico, respecto de las relaciones complejas en el sistema mundo, el crimen de agresión definido en Kampala se enfrenta a la oposición de los cinco Estados miembros del Consejo de Seguridad, en tanto ven limitado su derecho al uso de la fuerza contra otros países, enunciado en la Carta de la onU. Esta posición privilegiada se ha convertido en uno de los obstáculos más grandes para el futuro desarrollo, entrada en vigor y aplicabilidad del crimen de agresión, por parte de la CPI. Por ejemplo, Estados Unidos, que participó como Estado observador en Kampala, se opone ${ }^{22}$ a la entrada en vigencia del crimen de agresión en cabeza, si bien no exclusiva, de la CPI.

A esto se le debe sumar que la Corte Penal se encuentra limitada en su capacidad de investigar los crímenes que le competen, según lo consagrado en el Artículo 16 del ER:

21 K. Ambos, Óp. cit., p. 163.

22 Situación que contrasta con la imposición de los tribunales ad hoc para juzgar a líderes de países que han perdido su apoyo. Así por ejemplo, el último tribunal ad hoc fue impuesto por EE UU. como Estado invasor para juzgar a Sadam Hussein. Lo que ha sido considerado por los doctrinantes como una guerra de agresión. Igualmente, es de anotar que cuando a este país le interesa no veta las resoluciones para que el Consejo de Seguridad remita algunos casos a la Corte Penal, tal como sucedió con la Resolución 1593 sobre la situación en Darfur; tampoco vetó la Resolución 1973 de 2011, del Consejo de Seguridad sobre invasión a Libia. 
"En caso de que el Consejo de Seguridad, de conformidad con una resolución aprobada con arreglo a lo dispuesto en el Capítulo VII de la Carta de las Naciones Unidas, pida a la Corte que no inicie o que suspenda por un plazo de doce meses la investigación o el enjuiciamiento que haya iniciado, la Corte procederá a esa suspensión; la petición podrá ser renovada por el Consejo de Seguridad en las mismas condiciones".

Esta suspensión de una investigación por parte de la Corte, a petición del Consejo de Seguridad, se presentó en la Resolución 1422 de junio 12 de 2002, la cual fue prorrogada ${ }^{23}$ un año más a solicitud del Consejo de Seguridad, por la Resolución 1487 del 12 de julio de 2003. Dice así la Resolución 1422:

"Actuando con arreglo al Capitulo VII de la Carta de las Naciones Unidas, 1. Pide, de conformidad con lo dispuesto en el artículo 16 del Estatuto de Roma, que la Corte Penal Internacional, si surge un caso en relación con acciones $u$ omisiones relacionadas con operaciones establecidas o autorizadas por las Naciones Unidas y que entrañe la participación de funcionarios, ex funcionarios, personal o antiguo personal de cualquier Estado que no sea parte en el Estatuto de Roma y aporte contingentes, no inicie ni prosiga, durante un período de doce meses a partir del $1^{\circ}$ de julio de 2002, investigaciones o enjuiciamiento de ningún caso de esa índole salvo que el Consejo de Seguridad adopte una decisión en contrario;

2. Expresa la intención de renovar en las mismas condiciones, el $1^{\circ}$ de julio de cada año, la petición que se indica en el párrafo 1 para períodos sucesivos de doce meses durante el tiempo que sea necesario;

3. Decide que los Estados Miembros no tomarán ninguna medida que no esté en consonancia con el párrafo 1 y con sus obligaciones de carácter internacional.

4. Decide seguir ocupándose del asunto".

La resolución anterior es una facultad otorgada a las fuerzas de paz autorizadas por el Consejo de Seguridad de las Naciones Unidas en zonas de conflicto internacional donde participan soldados estadounidenses que, en caso de cometer crímenes de competencia de la Corte, no pueden ser juzgados por esta.

23 En contra de las prórrogas se pronuncia Ambos quien afirma: "Afortunadamente, el segundo intento de loS EE UU de prorrogar esta resolución fracasó debido a la oposición de varios miembros del Consejo de Seguridad, entre ellos China, que consideraron inoportuna la renovación por su coincidencia con el escándalo del abuso de prisioneros en Irak". Ver: Temas de derecho penal internacional y europeo, 32 (Marcial Pons, Barcelona, 2006). 
Esta situación constituye un obstáculo para que la Corte pueda actuar ante crímenes de agresión en un futuro ${ }^{24}$. Una situación esperanzadora al respecto puede inferirse del comentario de Concepción Hernández, quien destaca como importante el dato de que "desde el 2004, el Consejo no ha renovado su decisión de ejercer la prerrogativa de suspender la jurisdicción de la Corte conforme al artículo $16 " 25$.

Ahora bien, en cuanto al ejercicio y puesta en práctica del crimen de agresión en el contexto internacional, puede decirse que este se enfrenta a un sistema internacional fragmentado; por ejemplo, por un lado se encuentran las potencias que más incurren en este tipo de crimen y que se oponen a la entrada en vigencia del crimen de agresión en competencia de la Corte, tales como Estados Unidos ${ }^{26}$, China $^{27}$, Rusia ${ }^{28}$. Es de anotar que los cinco países le apuestan a que sea el Consejo de Seguridad el que conserve la competencia sobre el crimen de agresión con sustento en el Artículo 39 de la Carta de la ONU.

24 Amnistía Internacional, Corte Penal Internacional. La elección de las opciones correctas, 11. En http://www.amnesty.org/es/library/asset/IOR40/008/2010/es/5bad58cc-e7a8-4f5cbf92-93f19d5085c1/ior400082010es.pdf (2010). Varios países.

25 C. Hernández Escobar, El apoyo a la Corte: ¿un proceso irreversible hacia la universalidad? en El futuro de la Corte Penal Internacional, hacia el X aniversario de la entrada en vigor del Estatuto de Roma, 78-90 (Institut Català Internacional per la Pau, Barcelona, 2012).

26 EE UU critica la aprobación de la Resolución RC/6 sobre el crimen de agresión en los siguientes términos: "Los Estados Unidos se asocian a la crítica cuestión de principio planteada en las intervenciones que acaban de realizar los gobiernos de Francia y Reino Unido en relación con la precedencia del Consejo de Seguridad en virtud del Artículo 39 de la Carta de las UN en la determinación de la existencia de un acto de agresión y en la responsabilidad primordial del Consejo respecto de los asuntos de paz y seguridad internacionales". CPI, Documento XV, Declaración de Estados observadores sobre el crimen de agresión, RC/9/11, Conferencia de Revisión de Kampala (julio, 2010).

27 Respecto de la aprobación de la Resolución RC/6 sobre el crimen de agresión, China hizo la siguiente declaración: "En opinión de la delegación china, los dos artículos anteriormente mencionados (esto es el art 15 bis y 15 ter de las enmiendas) no han logrado reflejar la idea que, por lo que respecta un acto de agresión, es necesario que el Consejo de Seguridad determine previamente la existencia de éste para que la Corte Penal Internacional pueda ejercer su competencia sobre el crimen de agresión. La existencia de un acto de agresión debería ser determinada por el Consejo de Seguridad", Óp. cit.

28 Rusia se manifestó en los siguientes términos: "Como en todo consenso, no todos los elementos de la decisión consensuada satisfacen a cada uno. En particular, no consideramos que la decisión consensuada a la que aqui se llegó refleje en su totalidad el sistema existente de mantenimiento de la paz y de la seguridad encabezado por el Consejo de Seguridad, ante todo y sobre todo en la esfera de las prerrogativas del Consejo de Seguridad a la hora de decidir la existencia de un acto de agresión. En cualquier caso, la decisión se ha tomado y continuaremos trabajando en ella", Ibídem. 
El otro bloque de países está integrado por países europeos como Alemania, Francia, España, Holanda y Bélgica, entre otros, que le apuestan a fortalecer la Corte Penal Internacional. Igualmente se encuentran en esta posición, los países de América Latina y el Caribe, a excepción de Colombia y Guatemala. Es de precisar que con respecto al crimen de agresión aprobado en Kampala, los países miembros de UnAsur, Costa Rica y Trinidad y Tobago se comprometieron a emprender la ratificación de la enmienda sobre el crimen de agresión ${ }^{29}$. Los Estados parte determinaron que la Corte podrá ejercer su jurisdicción cuando treinta Estados hayan ratificado la enmienda.

Hasta el momento ${ }^{30}$, siete países han ratificado la enmienda: el primer país fue Liechtenstein, seguido de Trinidad y Tobago, Estonia, Luxemburgo, Alemania, Botsuana y Samoa. Según afirma el Instituto Global para la Prevención de la Agresión, algunos Estados ya han implementado la definición del crimen de agresión de Kampala, pero todavía no han ratificado las enmiendas (por ejemplo Croacia y Eslovenia). La ratificación,

29 Ver Declaración Especial sobre la Primera Conferencia de Revisión del Estatuto de Roma, aprobada en la Reunión Extraordinaria del Consejo de Jefas y Jefes de Estado y de Gobierno de UNASUR (4 de mayo de 2010). El pronunciamiento es el siguiente: "Las Jefas y los Jefes de Estado y de Gobierno de la Unión de Naciones Suramericanas, teniendo presente que el Tratado Constitutivo de UNASUR, firmado en Brasilia el 23 de mayo de 2008, consagra el principio del irrestricto respeto a los derechos humanos universales, indivisibles e interdependientes, como una de las condiciones esenciales para la construcción de un futuro común de paz y prosperidad económica y social y el desarrollo de los pueblos: 1. Expresan su convencimiento de que la impunidad de los autores de los crímenes más graves de trascendencia internacional establecidos en el Estatuto de Roma de la Corte Penal Internacional es uno de los factores que pone en riesgo la estabilidad del orden internacional. 2. Destacan la trascendencia histórica de la primera Conferencia de Revisión del Estatuto de Roma de la Corte Penal Internacional, que tendrá lugar en Kampala, Uganda, del 31 de mayo al 11 de junio de 2010". En http://www.movimientos.org/es/albasi/show_text. php3\%3Fkey\%3D17270.

30 De acuerdo con el Instituto Global para la Prevención de la Agresión (2013), los siguientes Estados partes se encuentran actualmente trabajando de forma activa sobre la ratificación de las enmiendas sobre el crimen de agresión: Alemania, Argentina, Australia, Austria, Bélgica, Brasil, Botsuana, Chile, Costa Rica, Croacia, Chipre, República Checa, República Dominicana, Ecuador, Eslovenia, España, Estonia, Georgia, Grecia, Italia, Lesoto, Malta, los Países Bajos, Panamá, Perú, Portugal, Rumania, Senegal, Suiza y Uruguay. Mientras que en Finlandia, Ghana, Guatemala, Japón, Letonia y Venezuela el proceso está en las etapas iniciales. Ver http://crimeofaggression.info/documents/1/ Informe_de_Avances_sobre-ESP.pdf (20 de marzo de 2014). Por su parte, los gobiernos de Costa Rica, 30 de julio de 2012, y de Uruguay, 10 de abril de 2013, presentaron proyectos de ley a sus respectivos congresos, tendientes a que se apruebe en sus legislaciones internas la ratificación de las enmiendas de Kampala sobre el crimen de agresión. 
sin embargo, contribuiría a la activación del ejercicio de la competencia de la $\mathrm{CPI}^{31}$.

Como se ha mencionado, dentro de los países contrarios a concederle competencias a la Corte Penal se encuentra Estados Unidos, miembro del Consejo Permanente de la ONU, que en el año 2002 retiró la firma al ER. Este país siempre ha mantenido la posición de que el Consejo de Seguridad tenga la competencia para determinar si existe o no un acto de agresión de un Estado contra otro, por consiguiente, solo después de que actúe el Consejo de Seguridad podría la CPI analizar si existe algún tipo de responsabilidad penal individual.

Bajo este argumento, el Congreso estadounidense promulgó la American Service-Members Protection Act (ASPA, o Ley para la Protección del Personal de Servicio), por medio de la cual se impide la cooperación de los Estados con la CPI y se restringe la ayuda militar y económica a países que no se comprometan con Estados Unidos a no entregar sus funcionarios nacionales a la Corte Penal. Esta posición se sustenta en el Artículo 98 del ER:

"La Corte no dará curso a una solicitud de entrega o de asistencia en virtud de la cual el Estado requerido deba actuar en forma incompatible con las obligaciones que le imponga el derecho internacional con respecto a la inmunidad de un Estado o la inmunidad diplomática de una persona o un bien de un tercer Estado, salvo que la Corte obtenga anteriormente la cooperación de ese tercer Estado para la renuncia a la inmunidad".

Al respecto, el senador republicano Jesse Helms declaró: “Con el establecimiento de una Corte Penal Internacional permanente cada vez más cerca, la amenaza de que los soldados y oficiales estadounidenses puedan ser algún día aprehendidos, extraditados y juzgados por 'crímenes de guerra', es cada vez mayor". Bajo este tipo de presión, cerca de 43 Estados partes del Estatuto de Roma han firmado este tipo de tratados bilaterales de inmunidad, que les obliga a no entregar a la Corte Penal a soldados o

31 Instituto Global para la Prevención de la Agresión, Manual de ratificación e implementación de las enmiendas de Kamala al Estatuto de Roma, 145-156 (Universidad de Princeton, Princeton, 2012). 
funcionarios estadounidenses a la CPI acusados de crímenes de su competencia. Dentro de estos países se encuentran Colombia (que firmó el acuerdo en septiembre de 2003) Bolivia, Honduras, Panamá, República Dominicana y Guatemala. Otros 56 Estados han rechazado la firma de estos tratados, algunos de América del Sur y la mayoría europeos ${ }^{32}$.

Ahora bien, otro punto importante de resaltar es el contenido del Artículo 27 del ER, que prohíbe de manera literal otorgar inmunidad a individuos que cometan crímenes de competencia de la Corte. Por ejemplo, la Corte Penal, por medio de la Resolución de la ONU, ordenó el arresto de dos presidentes en ejercicio como Omar al Bashir, mandatario en ejercicio de Sudán, por crímenes de guerra y contra la humanidad cometidos en Darfur, y del presidente libio, Muamar al Gadafi. Lo que evidencia este artículo es que ningún cargo oficial exime de responsabilidad penal por crímenes de competencia de la Corte ${ }^{33}$.

Esta remisión de las situaciones de Darfur y Libia por parte del Consejo de Seguridad a la Corte Penal, envió un mensaje de aceptación implícita de colaboración entre los Estados del Consejo Permanente de la onu y la Corte Penal Internacional, o fue un juego de doble moral, como siempre sucede cuando hay interés de por medio de uno de los países miembros. En todo caso, cualquiera de estas interpretaciones es válida en el realismo de la interdependencia compleja por la que atraviesan los Estados.

32 Ver al respecto, M. Torres Pérez, The American Service Members Protection Act 2002, LIV Revista Española de Derecho Internacional, 2, 1004-1008 (2002).

33 El Art. 27 del ER dice: "1. El presente Estatuto será aplicable por igual a todos sin distinción alguna basada en el cargo oficial. En particular, el cargo oficial de una persona, sea Jefe de Estado o de Gobierno, miembro de un gobierno o parlamento, representante elegido o funcionario de gobierno, en ningún caso la eximirá de responsabilidad penal ni constituirá per se motivo para reducir la pena. 2. Las inmunidades y las normas de procedimiento especiales que conlleve el cargo oficial de una persona, con arreglo al derecho interno o al derecho internacional, no obstarán para que la Corte ejerza su competencia sobre ella". 


\section{CONCLUSIÓN}

Respecto del crimen de agresión puede decirse, de modo general, lo siguiente:

a) El balance de la Conferencia de Revisión de Kampala es muy alentador para la comunidad internacional y para el DIH, pues finalmente se tiene una definición consensuada sobre el crimen de agresión, mediante un tratado vinculante y no impuesto por medio de tribunales ad hoc.

b) La Corte Penal Internacional asume la competencia para investigar y decidir la existencia del crimen de agresión cometido por un Estado contra otro. Hace falta, por ende, que cada Estado ratifique la enmienda para que este norma entre en vigor y pueda ser aplicada contra quienes cometen el crimen de agresión con la colaboración de los líderes o jefes de Estado.

c) La entrada en vigor del crimen de agresión está supeditada a que treinta Estados ratifiquen la enmienda; de este compromiso por la paz mundial depende que la Corte pueda, en un futuro, ejercer su competencia de juzgar individuos que, por intermedio de su liderazgo y mando dentro de un Estado, cometan este tipo de crimen.

d) La entrada en vigor del crimen de agresión enviará un mensaje al mundo en general, en especial a Estados Unidos, China y Rusia sobre la importancia de consolidar un código penal universal autónomo e imparcial, con competencia para juzgar los crímenes de agresión.

e) La ratificación de este crimen estará supeditada a las presiones y negociaciones que lleven a cabo los Estados miembros del Consejo de Seguridad de la onu con los demás Estados parte comprometidos con la competencia de la Corte Penal Internacional. La incidencia de Estados Unidos es inevitable pero también puede ser contraproducente, si ejerce presión particular sobre otros países miembro, pues puede correr el riesgo de quedarse solo en el escenario internacional.

f) Prácticamente, la posición que tomen los Estados miembros del Consejo Permanente de la onu y de la Corte Penal 
Internacional como Francia e Inglaterra, respecto de aceptar o no aceptar la cláusula opting out de la competencia de la CPI, marcará la entrada en vigor del crimen de agresión. Igualmente, la postura de países como Alemania, Holanda, España, miembros de UNASUR, serán clave.

g) De todas formas se debe esperar como mínimo hasta 2017 para saber qué pasará en el escenario internacional respecto al crimen de agresión y conocer la posición que expresarán Estados Unidos, China y Rusia. La Corte se tendrá que preparar para este momento crucial y proponer diversas alternativas a los Estados partes; de ello depende que la entrada en vigencia del crimen de agresión se concrete. 


\section{BIBLIOGRAFÍA}

\section{Libros}

Ambos, K., Temas de derecho penal internacional y europeo, 32 (Marcial Pons, Barcelona, 2006).

Ambos, K., El crimen de agresión después de Kampala, 157 (Universidad Externado de Colombia, Bogotá D.C., 2012).

Salmón, E., El crimen de agresión después de Kampala: soberanía de los Estados y lucha contra la impunidad, 45 (Instituto de Democracia y Derechos Humanos de la Pontificia Universidad Católica del Perú, Lima, 2011).

\section{Contribuciones en Obras Colectivas}

Ambos, K., El derecho penal internacional en la encrucijada: de la imposición ad hoc a un sistema universal basado en un tratado internacional, en Future Perspectives on International Criminal Justice, 161 (C. Stahn \& Van Den Herlk, Eds., T.M.C. Asser Press, The Hague, 2010).

Hernández Escobar, C., El apoyo a la Corte: ¿Un proceso irreversible hacia la universalidad?, en El futuro de la Corte Penal Internacional, hacia el X aniversario de la entrada en vigor del Estatuto de Roma, 78-90 (Institut Català Internacional per la Pau, Barcelona, 2012).

Zapico, M., El crimen de agresión y la Corte Penal Internacional, en Derecho penal entre la guerra y la paz, 722-723 (A. Nieto Martín \& S. Manacorda, Eds., Universidad Castilla de la Mancha, Madrid, 2009).

\section{Artículos}

Andreas, P., Second Thoughts on the Crime of Aggression, 20 The European Journal of International Law, 4, 1117-1128 (2009).

Torres Pérez, M., The American Service Members Protection Act 2002, LIV Revista Española de Derecho Internacional, 2, 1004-1008 (2002).

Quesada Alcalá, C., El crimen de agresión como amenaza a la seguridad global, Cuadernos de estrategia, 160, 77-116 (2013).

\section{Fuentes Digitales}

Amnistía Internacional, Corte Penal Internacional. La elección de las opciones correctas, en www.amnesty.org/es/library/asset/IOR40/008/2010/es/5bad58cce7a8-4f5c-bf92-93f19d5085c1/ior400082010es.pdf (2010).

Instituto Global para la Prevención de la Agresión, Estado de ratificación e imple- 
mentación de las enmiendas de Kampala sobre el crimen de agresión, en http:// crimeofaggression.info/documents/1/Informe_de_Avances_sobre-ESP.pdf (21 de enero de 2014).

\section{Documentos}

CPI. Documento XV, Declaración de Estados observadores sobre el crimen de agresión, RC/9/11. Conferencia de Revisión de Kampala (julio, 2010).

Instituto Global para la Prevención de la Agresión, Manual de ratificación e implementación de las enmiendas de Kamala al Estatuto de Roma (Universidad de Princeton, Princeton, 2012).

UNASUR, Declaración Especial sobre la Primera Conferencia de Revisión del Estatuto de Roma, aprobada en la Reunión Extraordinaria del Consejo de Jefas y Jefes de Estado y de Gobierno, en http://www.movimientos.org/es/albasi/show_text. php3\%3Fkey\%3D17270 (4 de mayo de 2010). 